\title{
Analytical Solution of the Fractional Fredholm Integrodifferential Equation Using the Fractional Residual Power Series Method
}

\author{
Muhammed I. Syam \\ Department of Mathematical Sciences, UAE University, Al-Ain, UAE \\ Correspondence should be addressed to Muhammed I. Syam; m.syam@uaeu.ac.ae
}

Received 2 May 2017; Revised 20 June 2017; Accepted 9 July 2017; Published 9 August 2017

Academic Editor: Cemıl Tunç

Copyright (C) 2017 Muhammed I. Syam. This is an open access article distributed under the Creative Commons Attribution License, which permits unrestricted use, distribution, and reproduction in any medium, provided the original work is properly cited.

We study the solution of fractional Fredholm integrodifferential equation. A modified version of the fractional power series method (RPS) is presented to extract an approximate solution of the model. The RPS method is a combination of the generalized fractional Taylor series and the residual functions. To show the efficiency of the proposed method, numerical results are presented.

\section{Introduction}

Fractional Fredholm integrodifferential equations have several applications in sciences and engineering. The closed form of the exact solution of such problems is difficult to find and in most of the cases is not available. For this reason, researchers are looking for the numerical solutions of such problems. Irandoust-Pakchin and Abdi-mazraeh [1] used the variational iteration method for solving fractional integrodifferential equations with the nonlocal boundary conditions. Adomian decomposition method is used in $[2,3]$ while the homotopy perturbation method is used in $[4,5]$. Wazwaz [6-8] studied the Fredholm integral equations of the form

$$
u(x)=f(x)+\lambda \int_{a}^{b} K(x, t) u(t) d t
$$

where $a$ and $b$ are constants, $\lambda$ is a parameter, $u(x)$ is a smooth function as the discussion required, and $K(x, t) \in$ $C(\mathbb{R} \times[a, b])$ is the kernel. In this paper, we study the generalization of the above problem. We study the following class of fractional Fredholm integrodifferential equations of the form

$$
\begin{aligned}
D^{\alpha} u(x)=f(x)+\lambda \int_{a}^{b} K(x, t) u^{m}(t) d t, & \\
& 0<\alpha \leq 1, x \in \mathbb{R}, a \leq t \leq b,
\end{aligned}
$$

subject to

$$
u(a)=a_{0} .
$$

The fractional derivative in (2) is in the Caputo sense. If $\alpha=0$, we do not need the initial condition (3) and we return back to the problem which is discussed by Wazwaz [8]. In the following definition and theorem, we write the definition of Caputo derivative as well as the power rule which we are using in this paper. For more details on the geometric and physical interpretation for Caputo fractional derivatives, see [9].

Definition 1. For $m$ to be the smallest integer that exceeds $\alpha$, the Caputo fractional derivatives of order $\alpha>0$ are defined as

$$
\begin{aligned}
& D^{\alpha} u(x) \\
& = \begin{cases}\frac{1}{\Gamma(m-\alpha)} \int_{0}^{x}(x-\tau)^{m-\alpha-1} \frac{d^{m} u(\tau)}{d \tau^{m}} d \tau, & m-1<\alpha<m, \\
\frac{d^{m} u(x)}{d x^{m}}, & \alpha=m \in \mathbb{N} .\end{cases}
\end{aligned}
$$

Theorem 2. The Caputo fractional derivative of the power function satisfies

$$
\begin{aligned}
& D^{\alpha} x^{p} \\
& = \begin{cases}\frac{\Gamma(p+1)}{\Gamma(p-\alpha+1)} x^{p-\alpha}, & m-1<\alpha<m, p>m-1, p \in \mathbb{R}, \\
0, & m-1<\alpha<m, p \leq m-1, p \in \mathbb{N} .\end{cases}
\end{aligned}
$$


Definition 3. The Riemann-Liouville fractional integral operator of order $\alpha$ of $u(x) \in C_{\gamma}, \gamma>-1$, is defined as

$$
\begin{aligned}
& I^{\alpha} u(x) \\
& = \begin{cases}\frac{1}{\Gamma(\alpha)} \int_{0}^{x}(x-\tau)^{\alpha-1} u(\tau) d \tau, & 0 \leq m-1<\alpha<m, \\
u(x), & \alpha=m \in \mathbb{N},\end{cases}
\end{aligned}
$$

where $C_{\gamma}$ is the space of real functions $u(x), x \in \mathfrak{R}$, such that, for each $u(x)$, there exists a real number $\rho>\gamma$ such that $u(x)=x^{\rho} u_{1}(x)$ where $u_{1}(x) \in C(\Re)$.

In addition, $u(x) \in C_{\gamma}^{m}$ if $u^{(m)}(x) \in C_{\gamma}$ where $m \in \mathbb{N}$. We present the following definition and some properties of the fractional power series which are used in this paper. More details can be found in [10].

Definition 4. A power series expansion of the form

$$
\begin{gathered}
\sum_{m=0}^{\infty} c_{m}\left(x-x_{0}\right)^{m \alpha}=c_{0}+c^{1}\left(x-x_{0}\right)^{\alpha}+c^{2}\left(x-x_{0}\right)^{2 \alpha} \\
+\cdots,
\end{gathered}
$$

where $0 \leq m-1<\alpha \leq m$, is called fractional power series FPS about. $x=x_{0}$.

Theorem 5. Suppose that $f$ has a fractional FPS representation at $x=x_{0}$ of the form

$$
g(x)=\sum_{m=0}^{\infty} c_{m}\left(x-x_{0}\right)^{m \alpha}, \quad x_{0} \leq x<x_{0}+\beta .
$$

If $D^{m \alpha} g(x), m=0,1,2, \ldots$, are continuous on $\mathbb{R}$, then $c_{m}=$ $\left.D^{m \alpha} g\left(x_{0}\right)\right) / \Gamma(1+m \alpha)$.

Theorem 6. Let $u(x) \in C\left(\left[x_{0}, x_{0}+R\right)\right)$ and $D^{i \alpha} u(x) \in$ $C\left(\left(x_{0}, x_{0}+R\right)\right)$ for $i=0,1, \ldots, m+1$ where $0 \leq m-1<\alpha \leq m$. Then,

$$
\begin{aligned}
& I^{(m+1) \alpha} D^{(m+1) \alpha} u(x) \\
& =\frac{D^{(m+1) \alpha}(\omega)}{\Gamma((m+1) \alpha+1)}\left(x-x_{0}\right)^{(m+1) \alpha+1}, \\
& x_{0} \leq \omega \leq x<x_{0}+R .
\end{aligned}
$$

Theorem 7. Let $u(x) \in C\left(\left[x_{0}, x_{0}+R\right)\right), D^{i \alpha} u(x) \in C\left(\left(x_{0}, x_{0}+\right.\right.$ $R)$ ), and $D^{i \alpha} u(x)$ can differentiate $(m-1)$ with respect to $x$ for $i=0,1, \ldots, m+1$ where $0 \leq m-1<\alpha \leq m$. Then,

$$
\begin{aligned}
u(x)= & \sum_{k=0}^{m} \frac{D^{k \alpha}(\omega)}{\Gamma(k \alpha+1)}\left(x-x_{0}\right)^{k \alpha} \\
+ & \frac{D^{(m+1) \alpha}(\omega)}{\Gamma((m+1) \alpha+1)}\left(x-x_{0}\right)^{(m+1) \alpha+1}, \\
& x_{0} \leq \omega \leq x<x_{0}+R .
\end{aligned}
$$

Theorem 8. Let $\left|D^{(m+1) \alpha} u(x)\right| \in A$ on $x_{0} \leq x<s$ where $m-1<\alpha \leq m$. Then, the reminder $R_{m}$ satisfies

$$
\left|R_{m}\right| \leq \frac{A}{\Gamma((m+1) \alpha+1)}\left(x-x_{0}\right)^{(m+1) \alpha},
$$

$$
x_{0} \leq x<s
$$

This paper is organized as follows. A description of the modified fractional power series method (MFPS) for approximating the fractional Fredholm integrodifferential equations problem (2)-(3) is presented in Section 2. Several numerical examples are discussed in Section 3. Conclusions and closing remarks are given in Section 4.

\section{Algorithm of the MFPS Method}

Consider the following class of fractional Fredholm integrodifferential equations of the form

$$
u(x)=f(x)+\lambda \int_{a}^{b} K(x, t) u(t) d t, \quad 0<\alpha \leq 1
$$

subject to

$$
u(a)=a_{0}
$$

Using the MFPS method, the solution problem (2)-(3) can be written the fractional power series form as

$$
u(x)=\sum_{n=0}^{\infty} f_{n} \frac{(x-a)^{n \alpha}}{\Gamma(1+n \alpha)} .
$$

To obtain the approximate values of the above series (14), the $k$ th truncated series $u_{k}(x)$ is written in the form

$$
u_{k}(x)=\sum_{n=0}^{k} f_{n} \frac{(x-a)^{n \alpha}}{\Gamma(1+n \alpha)}
$$

Since $u(a)=f_{0}=a_{0}$, we rewrite (14) as

$$
u_{k}(x)=a_{0}+\sum_{n=1}^{k} f_{n} \frac{(x-a)^{n \alpha}}{\Gamma(1+n \alpha)}, \quad k=1,2, \ldots
$$

where $u_{0}(x)=a_{0}$ is considered to be the 0th MRPS approximate solution of $u(x)$. To find the values of the MFPS-coefficients $f_{k}, k=1,2,3, \ldots$, we solve the fractional differential equation

$$
D^{(k-1) \alpha} \operatorname{Res}_{k}(u(a))=0, \quad k=1,2,3, \ldots,
$$

where $\operatorname{Res}_{k}(u(a))$ is the $k$ th residual function and is defined by

$$
\begin{aligned}
\operatorname{Res}_{k}(u(x))= & D^{\alpha} u_{k}(x)-f(x) \\
& -\lambda \int_{a}^{b} K(x, t) u_{k}(t) d t .
\end{aligned}
$$


To determine the coefficient $f_{1}$ in the expansion (15), we substitute the 1st RPS approximate solution

$$
u_{1}(x)=a_{0}+f_{1} \frac{(x-a)^{\alpha}}{\Gamma(1+\alpha)}
$$

into (18) to get

$$
\begin{aligned}
\operatorname{Res}_{1}(u(a)) \\
=\left(D^{\alpha} u_{1}(x)-f(x)-\lambda \int_{a}^{b} K(x, t) u_{1}(t) d t\right)_{x=a} \\
=f_{1}-f(a)-\lambda \int_{a}^{b} a_{0} K(a, t) d t \\
\quad-\lambda f_{1} \int_{a}^{b} \frac{(t-a)^{\alpha}}{\Gamma(1+\alpha)} K(x, t) d t=0 .
\end{aligned}
$$

Then, we solve $\operatorname{Res}_{1}(a)=0$ to get

$$
f_{1}=\frac{f(a)+\lambda \int_{a}^{b} a_{0} K(a, t) d t}{1-\lambda \int_{a}^{b}\left((t-a)^{\alpha} / \Gamma(1+\alpha)\right) K(a, t) d t} .
$$

To find $f_{2}$, we substitute the 2nd RPS approximate solution

$$
u_{2}(x)=a_{0}+f_{1} \frac{(x-a)^{\alpha}}{\Gamma(1+\alpha)}+f_{2} \frac{(x-a)^{2 \alpha}}{\Gamma(1+2 \alpha)}
$$

into the 2 nd residual function $\operatorname{Res}_{2}(u(x))$ such that

$$
\begin{gathered}
\operatorname{Res}_{2}(u(x))=D^{\alpha} u_{2}(x)-f(x)-\lambda \int_{a}^{b} K(x, t) \\
\cdot u_{2}(t) d t=f_{1}+f_{2} \frac{(x-a)^{\alpha}}{\Gamma(1+\alpha)}-f(x) \\
-\lambda \int_{a}^{b} K(x, t) \\
\cdot\left(a_{0}+f_{1} \frac{(t-a)^{\alpha}}{\Gamma(1+\alpha)}+f_{2} \frac{(t-a)^{2 \alpha}}{\Gamma(1+2 \alpha)}\right) d t .
\end{gathered}
$$

Then, we solve $D^{\alpha} \operatorname{Res}_{2}(u(a))=0$ to get

$$
f_{2}=\frac{D^{\alpha} f(a)+\lambda a_{0} \int_{a}^{b} D_{x}^{\alpha} K(a, t) d t+\lambda f_{1} \int_{a}^{b}\left((t-a)^{\alpha} / \Gamma(1+\alpha)\right) D_{x}^{\alpha} K(a, t) d t}{1-\lambda \int_{a}^{b}\left((t-a)^{2 \alpha} / \Gamma(1+2 \alpha)\right) D_{x}^{\alpha} K(x, t) d t} .
$$

To find $f_{3}$, we substitute the 3rd RPS approximate solution

$$
\begin{aligned}
u_{3}(x)= & a_{0}+f_{1} \frac{(x-a)^{\alpha}}{\Gamma(1+\alpha)}+f_{2} \frac{(x-a)^{2 \alpha}}{\Gamma(1+2 \alpha)} \\
& +f_{3} \frac{(x-a)^{3 \alpha}}{\Gamma(1+3 \alpha)}
\end{aligned}
$$

into the 3rd residual function $\operatorname{Res}_{3}(u(x))$ such that

$$
\operatorname{Res}_{3}(u(x))=D^{\alpha} u_{3}(x)-f(x)-\lambda \int_{a}^{b} K(x, t)
$$

$$
f_{3}=\frac{f_{2}+D^{2 \alpha} f(a)+\lambda \sum_{k=0}^{2} f_{k} \int_{a}^{b}\left((t-a)^{k \alpha} / \Gamma(1+k \alpha)\right) D_{x}^{2 \alpha} K(a, t) d t}{1-\lambda \int_{a}^{b}\left((t-a)^{3 \alpha} / \Gamma(1+3 \alpha)\right) D_{x}^{2 \alpha} K(a, t) d t} .
$$

$$
\begin{aligned}
& \cdot u_{3}(t) d t=f_{1}+f_{2} \frac{(x-a)^{\alpha}}{\Gamma(1+\alpha)}+f_{3} \frac{(x-a)^{2 \alpha}}{\Gamma(1+2 \alpha)} \\
& -f(x)-\lambda \int_{a}^{b} K(x, t)\left(a_{0}+f_{1} \frac{(t-a)^{\alpha}}{\Gamma(1+\alpha)}\right. \\
& \left.+f_{2} \frac{(t-a)^{2 \alpha}}{\Gamma(1+2 \alpha)}+f_{3} \frac{(t-a)^{3 \alpha}}{\Gamma(1+3 \alpha)}\right) d t .
\end{aligned}
$$

Then, we solve $D^{2 \alpha} \operatorname{Res}_{3}(u(a))=0$ to get
To find $f_{4}$, we substitute the 4 th RPS approximate solution

$$
\begin{aligned}
u_{4}(x)= & a_{0}+f_{1} \frac{(x-a)^{\alpha}}{\Gamma(1+\alpha)}+f_{2} \frac{(x-a)^{2 \alpha}}{\Gamma(1+2 \alpha)} \\
& +f_{3} \frac{(x-a)^{3 \alpha}}{\Gamma(1+3 \alpha)}+f_{4} \frac{(x-a)^{4 \alpha}}{\Gamma(1+4 \alpha)}
\end{aligned}
$$

into the 4 th residual function $\operatorname{Res}_{4}(u(x))$ such that

$$
\begin{array}{r}
\operatorname{Res}_{4}(u(x))=D^{\alpha} u_{4}(x)-f(x)-\lambda \int_{a}^{b} K(x, t) \\
\cdot u_{4}(t) d t=f_{1}+f_{2} \frac{(x-a)^{\alpha}}{\Gamma(1+\alpha)}+f_{3} \frac{(x-a)^{2 \alpha}}{\Gamma(1+2 \alpha)}
\end{array}
$$




$$
\begin{gathered}
+f_{4} \frac{(x-a)^{4 \alpha}}{\Gamma(1+4 \alpha)}-f(x)-\lambda \int_{a}^{b} K(x, t)\left(a_{0} \quad+f_{4} \frac{(x-a)^{4 \alpha}}{\Gamma(1+4 \alpha)}\right) d t . \\
+f_{1} \frac{(x-a)^{\alpha}}{\Gamma(1+\alpha)}+f_{2} \frac{(x-a)^{2 \alpha}}{\Gamma(1+2 \alpha)}+f_{3} \frac{(x-a)^{3 \alpha}}{\Gamma(1+3 \alpha)} \quad \text { Then, we solve } D^{3 \alpha} \operatorname{Res}_{4}(u(a))=0 \text { to get } \\
f_{4}=\frac{f_{3}+D^{3 \alpha} f(a)+\lambda \sum_{k=0}^{3} f_{k} \int_{a}^{b}\left((t-a)^{k \alpha} / \Gamma(1+k \alpha)\right) D_{x}^{2 \alpha} K(a, t) d t}{1-\lambda \int_{a}^{b}\left((t-a)^{4 \alpha} / \Gamma(1+4 \alpha)\right) D_{x}^{2 \alpha} K(a, t) d t} .
\end{gathered}
$$

Using similar argument, we find that

$$
f_{n}=\frac{f_{n-1}+D^{(n-1) \alpha} f(a)+\lambda \sum_{k=0}^{n-1} f_{k} \int_{a}^{b}\left((t-a)^{k \alpha} / \Gamma(1+k \alpha)\right) D_{x}^{(n-1) \alpha} K(a, t) d t}{1-\lambda \int_{a}^{b}\left((t-a)^{n \alpha} / \Gamma(1+n \alpha)\right) D_{x}^{(n-1) \alpha} K(a, t) d t}, \quad n=1,2,3, \ldots
$$

Thus,

$$
u_{k}(x)=a_{0}+\sum_{n=1}^{k}\left(\frac{f_{n-1}+D^{(n-1) \alpha} f(a)+\lambda \sum_{k=0}^{n-1} f_{k} \int_{a}^{b}\left((t-a)^{k \alpha} / \Gamma(1+k \alpha)\right) D_{x}^{(n-1) \alpha} K(a, t) d t}{1-\lambda \int_{a}^{b}\left((t-a)^{n \alpha} / \Gamma(1+n \alpha)\right) D_{x}^{(n-1) \alpha} K(a, t) d t}\right) \frac{(x-a)^{n \alpha}}{\Gamma(1+n \alpha)}
$$

For $k=1,2, \ldots$

\section{Numerical Results}

In this section, we present three examples to show the efficiency of the proposed method. We use Mathematica software to generate the results in this section.

Example 1. Consider the following fractional Fredholm integrodifferential equation:

$$
D^{1 / 2} u(x)=\frac{32}{3 \sqrt{\pi}} x^{1.5}+\frac{16}{3 \sqrt{\pi}} x^{2.5}-2 x+\int_{0}^{1} x t u(t)
$$

$$
x \in \mathbb{R}
$$

subject to

$$
u(0)=0
$$

The exact solution is

$$
u(x)=4 x^{2}+5 x^{3}
$$

Using the same argument described in the previous section, we find that

$$
\begin{aligned}
& f_{0}=f_{1}=f_{2}=f_{3}=0, \\
& f_{4}=8,
\end{aligned}
$$

$$
\begin{aligned}
& f_{5}=0, \\
& f_{6}=30, \\
& f_{n}=0, \quad n=7,8, \ldots
\end{aligned}
$$

Thus,

$$
u_{6}(x)=4 x^{2}+5 x^{3}
$$

which is the exact solution.

Example 2. Consider the following fractional Fredholm integrodifferential equation:

$$
\begin{aligned}
D^{1 / 4} u(x)= & \sum_{k=0}^{\infty} \frac{x^{k+3 / 4}}{2^{k+1} \Gamma(k-1 / 4)}-(4-2 \sqrt{e}) x^{2} \\
& +\int_{0}^{1} x^{2} t u(t), \quad x \in \mathbb{R}
\end{aligned}
$$

subject to

$$
u(0)=1
$$

The exact solution is

$$
u(x)=e^{(1 / 2) x}
$$


Using the same argument described in the previous section, we find the first few terms which are

$$
\begin{aligned}
& f_{0}=1, \\
& f_{1}=f_{2}=f_{3}=0, \\
& f_{4}=\frac{1}{2}, \\
& f_{5}=f_{6}=f_{7}=0, \\
& f_{8}=\frac{1}{4} .
\end{aligned}
$$

Continuing in this process, we find that

$$
f_{n}= \begin{cases}\frac{1}{2^{k}}, & n=4 k, k=0,1,2, \ldots \\ 0, & \text { otherwise }\end{cases}
$$

Thus, if $n=4 k$ for some positive integer $k$,

$$
\begin{aligned}
u_{n}(x) & =\sum_{m=0}^{n} f_{m} \frac{x^{m / 4}}{\Gamma(1+m / 4)}=\sum_{m=0}^{k} \frac{1}{2^{m}} \frac{x^{4 m / 4}}{\Gamma(1+4 m / 4)} \\
& =\sum_{m=0}^{k} \frac{1}{2^{m}} \frac{x^{m}}{m !} .
\end{aligned}
$$

Hence,

$$
\lim _{n \rightarrow \infty} u_{n}(x)=\lim _{n \rightarrow \infty} \sum_{m=0}^{k} \frac{1}{2^{m}} \frac{x^{m}}{m !}=\sum_{m=0}^{\infty} \frac{1}{2^{m}} \frac{x^{m}}{m !}=e^{(1 / 2) x}
$$

which is the exact solution.

Example 3. Consider the following fractional Fredholm integrodifferential equation:

$$
D^{1 / 3} u(x)=\frac{\Gamma(4 / 3)}{\Gamma(11 / 3)}-5 e^{x}+4 \int_{0}^{1} e^{x} t u(t) d t,
$$

$$
x \in \mathbb{R}
$$

subject to

$$
u(0)=1 .
$$

The exact solution is

$$
u(x)=1+x^{3} .
$$

Using the same argument described in the previous section, we find that the first few terms are

$$
\begin{aligned}
& f_{0}=1, \\
& f_{1}=f_{2}=f_{3}=f_{4}=0, \\
& f_{5}=f_{6}=f_{7}=f_{8}=0, \\
& f_{9}=6, \\
& f_{9}=f_{10}=0 .
\end{aligned}
$$

Continuing in this process, we find that

$$
f_{n}= \begin{cases}1, & n=0 \\ 6, & n=9 \\ 0, & \text { otherwise }\end{cases}
$$

Thus,

$$
u_{9}(x)=1+x^{3}
$$

which is the exact solution.

\section{Conclusions and Closing Remarks}

In this paper we employed the MFPS method to handle the fractional Fredholm integrodifferential problems. The method showed reliability in handling these ill-posed problems. It is worth mentioning that we get the exact solution in the above three examples. We test the prosed method and we get very accurate results. Although the MRPS method is not commonly used for such problems, it gives us very accurate results. This technique can be extended to other applications in science and engineering.

\section{Conflicts of Interest}

The author declares that there are no conflicts of interest regarding the publication of this paper.

\section{Acknowledgments}

The author also would like to express his sincere appreciation to the United Arab Emirates University Research Affairs for the financial support of Grant no. SURE Plus 21.

\section{References}

[1] S. Irandoust-Pakchin and S. Abdi-mazraeh, "Exact solutions for some of the fractional integro-differential equations with the nonlocal boundary conditions by using the modification of He's variational iteration method," International Journal of Advanced Mathematical Sciences, vol. 1, no. 3, pp. 139-144, 2013.

[2] S. Saha Ray, "Analytical solution for the space fractional diffusion equation by two-step Adomian decomposition method," Communications in Nonlinear Science and Numerical Simulation, vol. 14, no. 4, pp. 1295-1306, 2009.

[3] R. C. Mittal and R. Nigam, "Solution of fractional integrodifferential equations by Adomain decomposition method," International Journal of Applied Mathematics and Mechanics, vol. 4, no. 2, pp. 87-94, 2008.

[4] H. Saeedi and F. Samimi, "He's homotopy perturbation method for nonlinear ferdholm integrodifferential equations of fractional order," International Journal of Engineering Research and Applications, vol. 2, no. 5, pp. 52-56, 2012.

[5] R. K. Saeed and H. M. Sdeq, "Solving a system of linear fredholm fractional integro-differential equations using homotopy perturbation method," Australian Journal of Basic and Applied Sciences, vol. 4, no. 4, pp. 633-638, 2010. 
[6] A. M. Wazwaz, A First Course in Integral Equations, New Jersey, NJ, USA, WSPC, 1997.

[7] A. Wazwaz, Partial Differential Equations and Solitary Waves Theory, Higher Education Press, Beijing, China; Springer, Berlin, Germany, 2009.

[8] A.-M. Wazwaz, "The regularization method for Fredholm integral equations of the first kind," Computers \& Mathematics with Applications. An International Journal, vol. 61, no. 10, pp. 2981-2986, 2011.

[9] M. Caputo, "Linear models of dissipation whose $Q$ is almost frequency independent-II," Geophysical Journal of the Royal Astronomical Society, vol. 13, no. 5, pp. 529-539, 1967.

[10] O. Abu Arqub, A. El-Ajou, A. S. Bataineh, and I. Hashim, "A representation of the exact solution of generalized Lane-Emden equations using a new analytical method," Abstract and Applied Analysis, vol. 2013, Article ID 378593, 10 pages, 2013. 


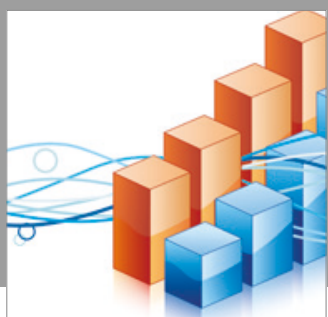

Advances in

Operations Research

vatersals

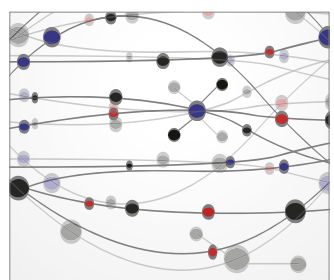

\section{The Scientific} World Journal
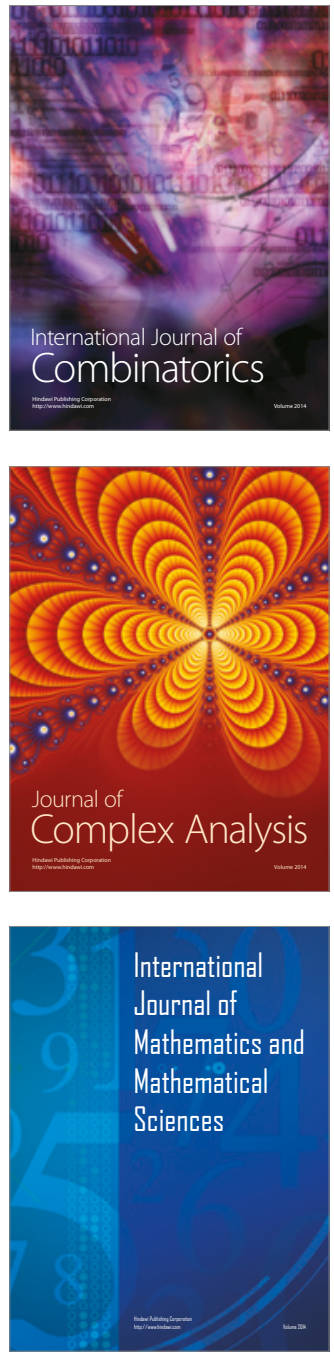
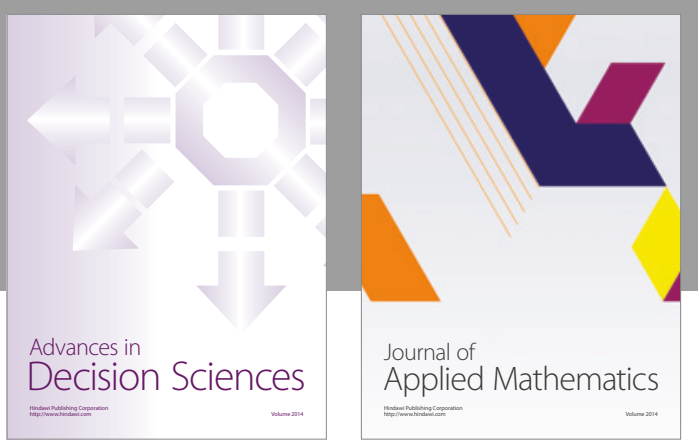

Algebra

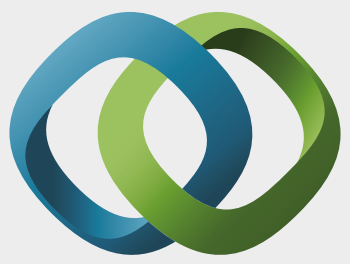

\section{Hindawi}

Submit your manuscripts at

https://www.hindawi.com
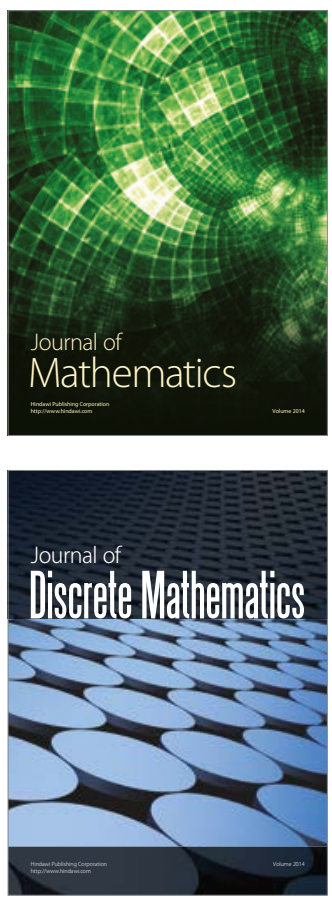

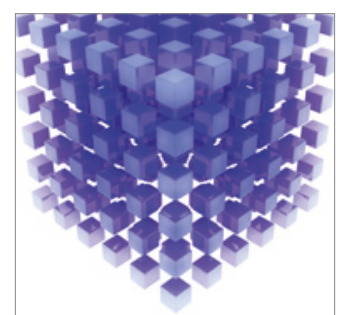

Mathematical Problems in Engineering
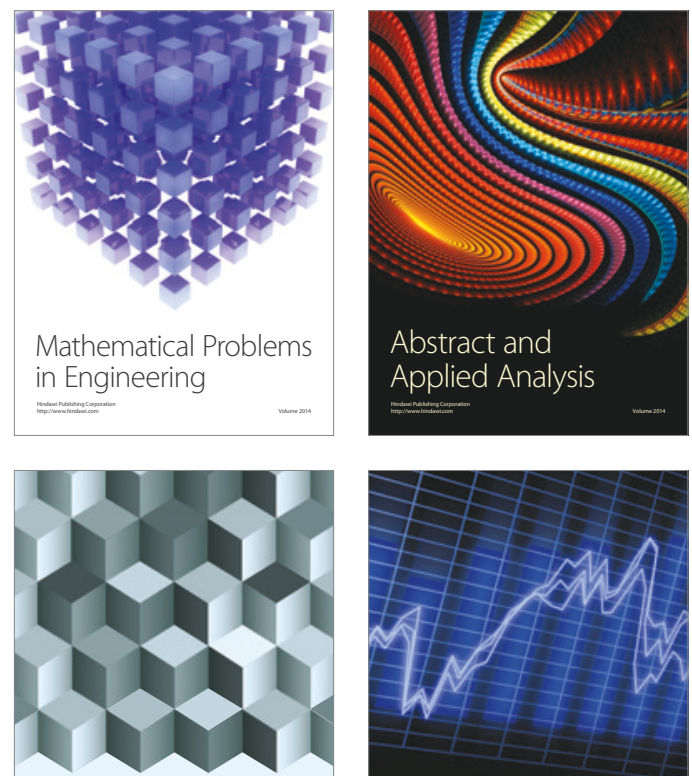

Journal of

Function Spaces

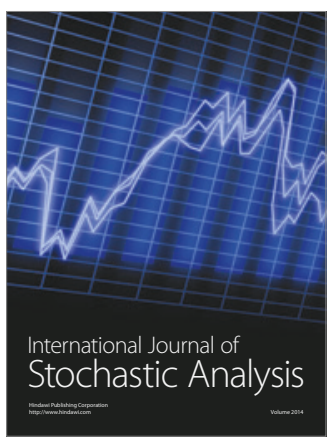

Probability and Statistics
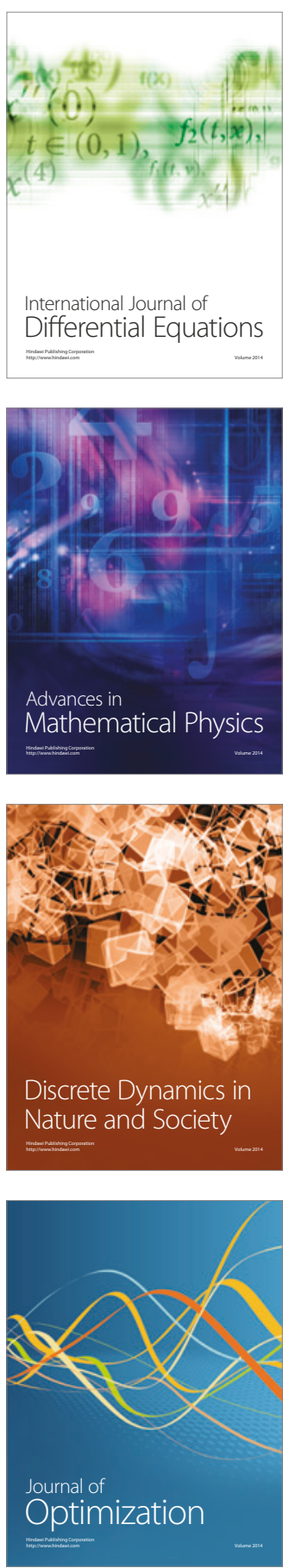